\title{
Lacrimal Diversion Devices (Sinopsys Lacrimal Stent): Sharing our Experience with Patients with Chronic Rhinosinusitis without Polyposis
}

\author{
Peter Baptista ${ }^{1}$ Octavio Garaycochea ${ }^{1}$ Carlos Prieto-Matos ${ }^{1} \quad$ Marta Alvarez de Linera Alperi ${ }^{1 \odot}$ \\ Juan Alcalde ${ }^{1}$ \\ ${ }^{1}$ Department of Otorhinolaryngology, Clinica Universitaria de \\ Navarra, Pamplona, Spain \\ Int Arch Otorhinolaryngol 2019;23:e422-e426. \\ Address for correspondence Marta Alvarez de Linera Alperi, \\ Department of Otorhinolaryngology, Clinica Universitaria de Navarra, \\ Avenida Pio XII 36, 31008, Pamplona 31008, Spain \\ (e-mail: malvarezdel@unav.es).
}

\begin{abstract}
Keywords

- sinusitis

- paranasal sinuses

- nasolacrimal duct

- minimally invasive surgical procedures

- nasal surgical procedures

- drug delivery systems
\end{abstract}

Introduction Chronic rhinosinusitis (CRS) is a highly prevalent pathology in our society. Due to the prevalence of this condition and to the persisting symptoms despite an appropriate medical treatment, surgical techniques are often required. Lately, minimal invasive techniques have been described, such as lacrimal diversion devices (LDDs). This technique offers a fast and convenient choice for delivery of sinus irrigation and topical medication.

Objective We aimed to describe our experience with LDDs and evaluate the safety and effectiveness of the procedure in patients with moderate to severe CRS without nasal polyposis (CRSsNP) and persistent symptomatology despite medical therapy.

Methods A total of 7 patients underwent bilateral lacrimal stents placement in the operating room. A retrospective observational study was conducted. The Sino-Nasal Outcome Test-20 (SNOT-20) survey was performed and the score obtained was compared before and 1 month after the procedure.

Results The LDDs were used for an average of 80 days. During the follow-up, only three patients had a mild complication with the device (granuloma in the punctum, obstruction, and early extrusion). The mean baseline SNOT-20 score dropped significantly $(p=0.015)$ from 25.85 to 11.57 (mean: - 14.29) 1 month after the procedure.

Conclusion According to our experience and results, the use of LDD is a novel, feasible, and less invasive technique to treat refractory CRS. It reduces the risk of mucosal stripping, provides short-term outcomes, and the surgical procedure does not require advanced training in endoscopic sinus surgery. Moreover, it can be performed in-office under local anesthesia or sedation.

\section{Introduction}

Chronic rhinosinusitis (CRS) in adults is defined as a sinonasal inflammation persisting for $>12$ weeks characterized by nasal obstruction/congestion/blockage, nasal drainage (mucopurulent), facial pain/pressure/fullness, and decreased or loss of sense of smell. ${ }^{1,2}$ It is a highly prevalent condition, affecting $\sim 12$ to $15.2 \%$ of the adult population in the United States, exceeding other common respiratory conditions such

received September 12, 2018 accepted

February 1, 2019
DOI https://doi.org/ 10.1055/s-0039-1683849. ISSN 1809-9777. as acute asthma or chronic bronchitis. ${ }^{2}$ It has a direct impact on the overall quality of life and well-being of the patient, ${ }^{3}$ as well as on the health care system; the overall direct cost burden of CRS in the United States has been estimated at US\$ 8.6 billion per year. ${ }^{4}$

Surgery for CRS is generally indicated when symptoms persist despite medical therapy. Functional endoscopic sinus surgery (FESS) can improve the symptoms in
Copyright $\odot 2019$ by Thieme Revinter Publicações Ltda, Rio de Janeiro, Brazil
License terms

(1) $\Theta \circledast$ 
$98.4 \%$ of the patients at 7.8 years of follow-up. ${ }^{5}$ The efficacy of FESS can be attributable to the restoration of the ventilation and to the drainage of the paranasal sinuses by enlarging the narrowed or obstructed sinus ostia, and also by improving the topical delivery of medications to the sinonasal mucosa. ${ }^{6}$ Surgical techniques have evolved from open approaches to endoscopic ones, and, lately, minimal invasive tissue-sparing techniques have been described.

Minimally invasive techniques have been categorized as minimally invasive sinus technique (MIST), which include all procedures directed at the spaces around the sinus ostia avoiding the sinus ostia, such as the ethmoid punch sinusotomy (EPS), ${ }^{7,8}$ and the balloon catheter dilatation (BCD) sinusotomy, which directly dilate the natural ostia of the large sinuses-the maxillary, frontal and sphenoid. The anterior and posterior ethmoid sinuses cannot be directly addressed with BCD. ${ }^{8}$

The used of lacrimal diversion devices (LDDs) is a novel and alternative technique for the delivery of sinus irrigation of the anterior ethmoid-frontal recess area. The LDD stent is a flexible polymeric conduit designed to maintain a patent lumen between the lateral aspect of the caruncle and the paranasal sinus via an osteotomy in the lacrimal bone. (The Sinopsys Lacrimal Stent [Synopsys Surgical, Inc., Boulder, CO, USA] is currently an investigational device in the United States for sinus irrigation and is commercially available in the European Union under the CE Mark) This procedure allows the sinus irrigation and topical delivery of medication via a transcaruncular-ethmoid sinus route. A cadaver study has shown that delivering irrigation to the anterior ethmoid region is a feasible technique that can be used as a potential alternate technique for the delivery of sinus irrigation. $^{9}$

The objective of the present study is to describe our experience with LDDs and to evaluate the safety and effectiveness of the procedure in patients with CRS without nasal polyposis (CRSsNP). All of the patients were previously treated with medical therapy without a complete response to the treatment and were candidates for procedural intervention.

\section{Materials and Methods}

After providing informed consent, 7 patients ( $>18$ years old) with moderate to severe CRSsNP who had persistent symptoms despite medical therapy ( 1 course of oral antibiotics, 9-day taper of oral corticoids, 3 months of topical fluticasone and saline nasal irrigations) underwent bilateral lacrimal stent placement in the operating room. A standard sinus computed tomography (CT) was previously performed to evaluate to disease and to determine the location and the approximate trajectory for the placement of the LDD. Patients underwent planned turbinoplasty, septoplasty, or additional sinus procedures as deemed required. The patients were discharged with a cycle of 10 days of ophthalmic drops of corticosteroids / antibiotics alternated with saline drops.

\section{Surgical Procedure}

The placement of LDDs was performed following the recommendations of the manufacturer. The insertion is performed in a supine position. An eyelid retractor and a corneal protector are placed first, then a $25 \mathrm{G}$ needle is inserted in the region of the caruncle, and a local anesthetic is infiltrated (lidocaine hydrochloride and epinephrine).Afterwards, a $21 \mathrm{G} \times 1$-inch needle mounted on a 5 -ml saline-filled syringe is inserted behind and inferiorly to the caruncle, and the needle is advanced in a lateral to medial and caudal trajectory to encounter the bony junction between the medial orbit and the lacrimal fossa just anterior to the superior aspect of the posterior lacrimal crest. It passes between the superior and inferior canaliculi and posterior and superior to the common canaliculus. The needle is advanced through the lacrimal bone perpendicular to the surface of the bone; aspiration and observation of air flash in the syringe confirms the placement. A guidewire is placed through the needle lumen using the Seldinger technique ( $\mathbf{- F i g . 1}$ ). The $21 \mathrm{G}$ needle is removed and the obturator with cannula is inserted; subsequently, the obturator is removed and a trephine is inserted to enlarge the osteotomy (-Fig. 2). Finally, the lacrimal stent delivery instrument is advanced over the guidewire into proper placement. The guidewire and sheath are then removed.

\section{Statistical Analysis and Outcome Measures}

A retrospective observational study of patients who underwent LDD placement in our center was performed. To assess the impact and to measure the outcome of the technique, the Sino-Nasal Outcome Test-20 (SNOT-20) survey was performed before and 1 month after the procedure. The student t-test was used to compare variables, and $p<0.05$ was considered statistically significant.

\section{Results}

A total of 7 patients who underwent LDD placement where included ( 3 females, 4 males), with a mean age of 52.71 years old. The average preprocedure SNOT-20 score was 25.85 (-Table 1). In all of the patients, the procedure was performed under general anesthesia, and the LDDs were correctly placed without any complications during the surgery. All of the patients required an additional intervention: $71 \%$ underwent concomitant septoplasty, 14\% a turbinoplasty, and $85 \%$ required a concomitant sinus procedure (sinus osteotomy or balloon sinuplasty)

The average time the LDDs were used was 80 days (range: 6-124 days) (-Table 1). In the follow-up, one patient had a device-related mild complication, a granuloma in the punctum that was self-limited; in one patient the stents suffered from an obstruction and were therefore removed; and one patient had an early extrusion of the stents (-Table 2 ).

The SNOT-20 scores at baseline and at 1 month after the surgery for all patients are shown in - Fig. 3. The reduction observed in the score was clinically and statistically significant $(p=0.015)$, with a mean reduction of -14.29 (standard deviation [SD]: 11.25) (-Table 1). 


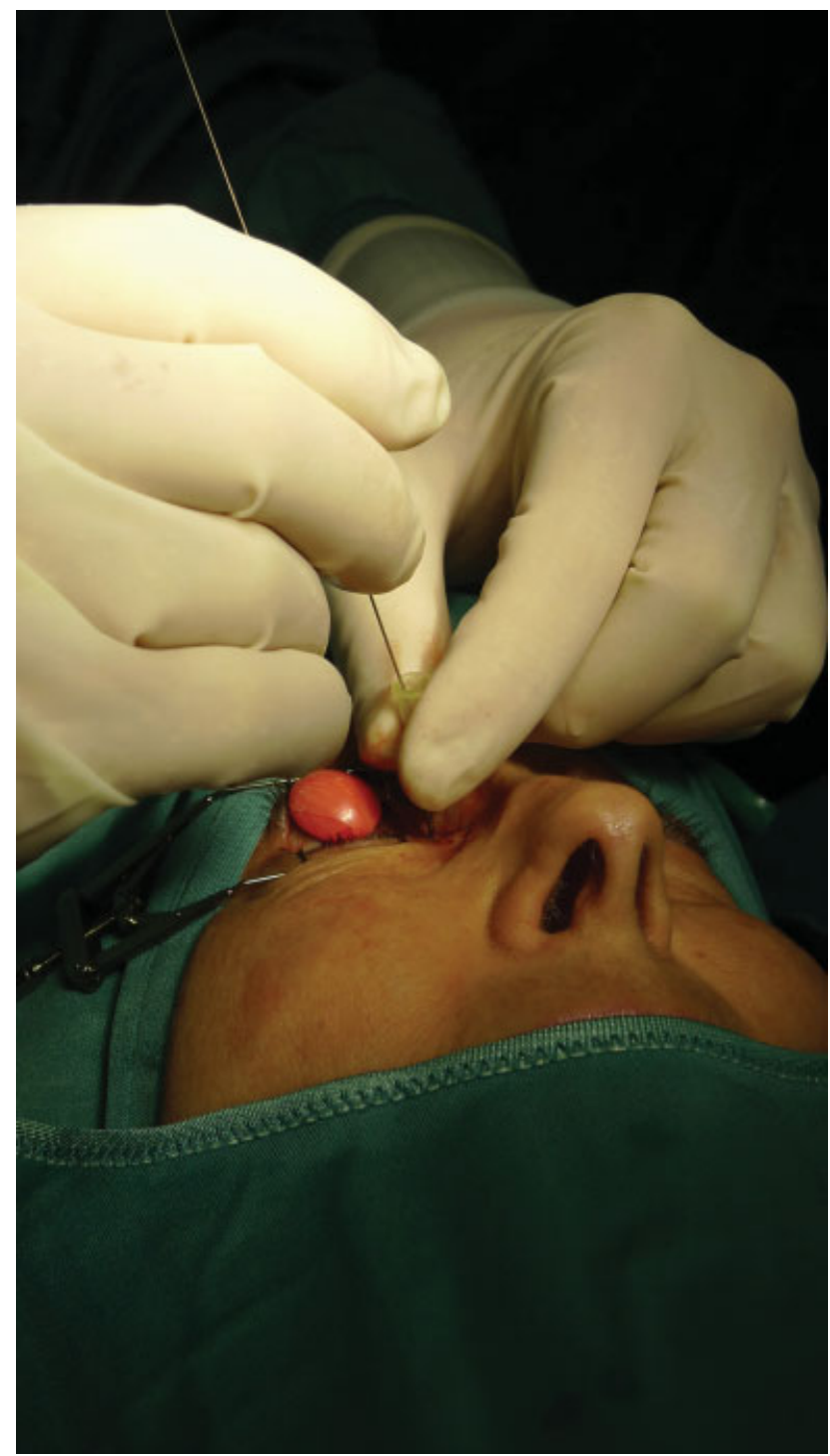

Fig. 1 Using the Seldinger technique, place the guidewire through and down the lumen of the needle. Apply gentle finger pressure on the guidewire to maintain position in the anterior ethmoid or in the nasal cavity while removing the needle.

Table 1 Outcomes of the Sino-Nasal Outcome Test -20

\begin{tabular}{|c|c|c|c|c|}
\hline & Time Point & & & $p$-value \\
\hline & Baseline & Postoperative & $\begin{array}{l}\text { Change } \\
\text { from } \\
\text { baseline }\end{array}$ & \\
\hline \multicolumn{4}{|c|}{ Patient } & \\
\hline 1 & 14 & 3 & 11 & \\
\hline 2 & 24 & 2 & 22 & \\
\hline 3 & 17 & 8 & 9 & \\
\hline 4 & 14 & 8 & 6 & \\
\hline 5 & 77 & 45 & 32 & \\
\hline 6 & 28 & 7 & 21 & \\
\hline 7 & 7 & 8 & 1 & \\
\hline $\begin{array}{l}\text { Mean } \\
\text { (SD) }\end{array}$ & $\begin{array}{l}25.85 \\
(23.59)\end{array}$ & $11.57(14.95)$ & $\begin{array}{l}14.29 \\
(11.25)\end{array}$ & 0.015 \\
\hline
\end{tabular}

Abbreviations: SD, standard deviation.

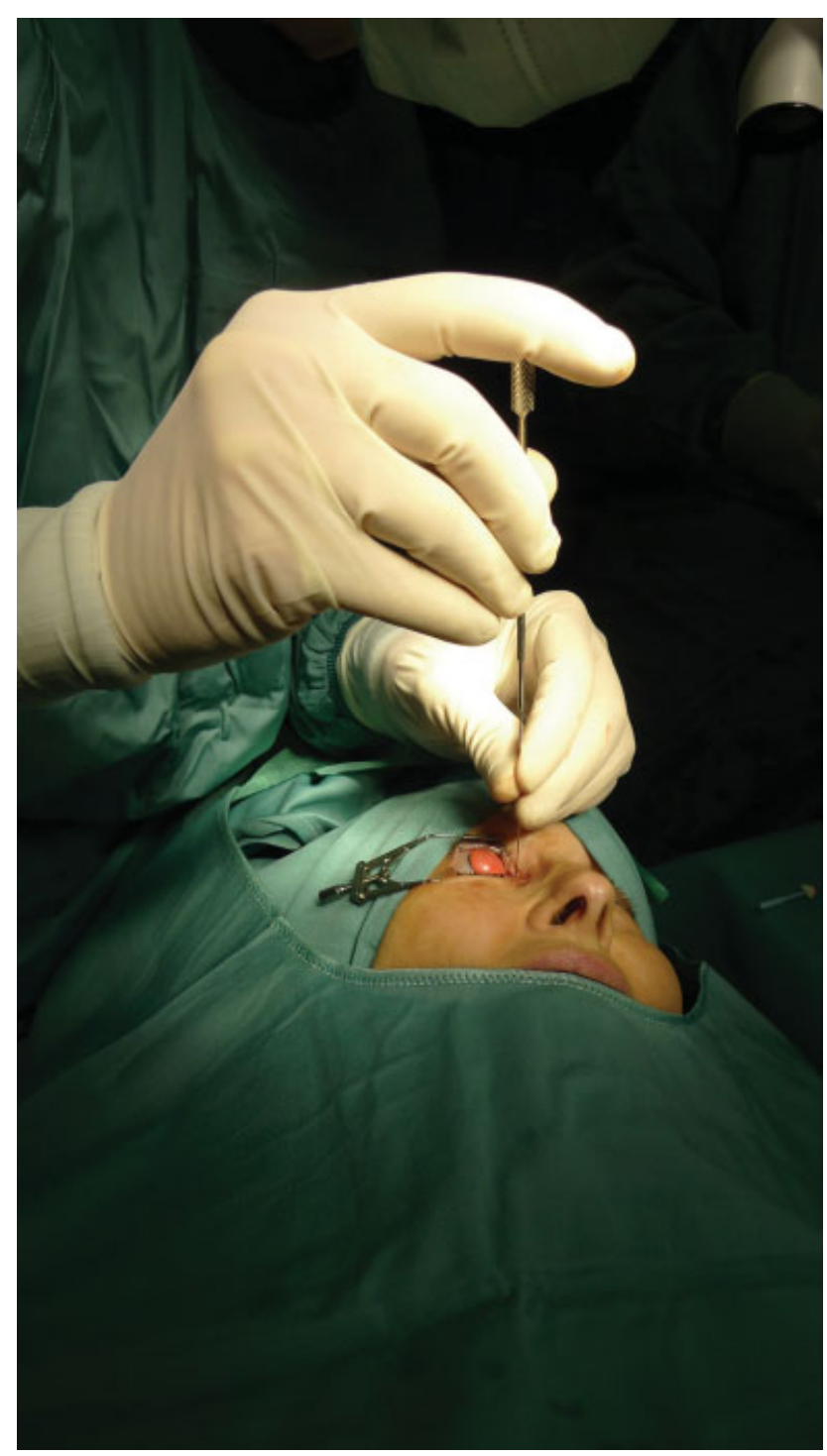

Fig. 2 While maintaining the placement of the guidewire, remove the trephine with a gently reversed twisting motion and then remove the cannula.

Table 2 Time with lacrimal diversion device and complications

\begin{tabular}{|l|l|l|}
\hline Patients & $\begin{array}{l}\text { Time with } \\
\text { LDD (days) }\end{array}$ & Complications \\
\hline 1 & 98 & - \\
\hline 2 & 40 & - \\
\hline 3 & 121 & - \\
\hline 4 & 115 & Obstruction \\
\hline 5 & 124 & - \\
\hline 6 & 61 & Granuloma (punctum) \\
\hline 7 & 6 & Extrusion \\
\hline Mean (SD) & $80(45.82)$ & \\
\hline
\end{tabular}

Abbreviations: LDD, lacrimal diversion device; SD, standard deviation. 


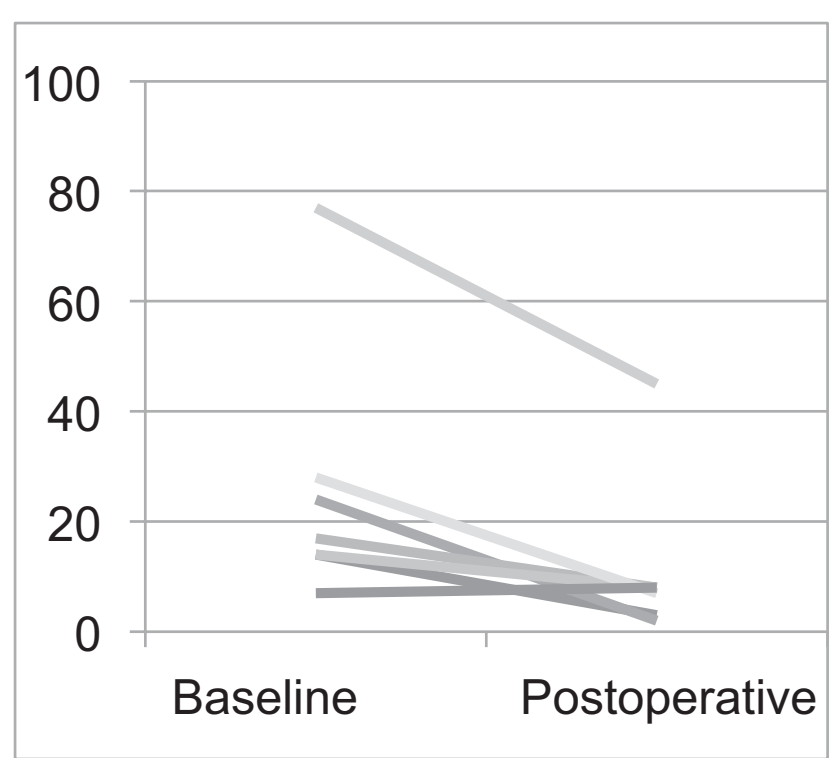

Fig. 3 Baseline and postoperative Sino-Nasal Outcome Test -20 (SNOT-20).

\section{Discussion}

Nowadays, FESS is considered a highly effective technique when addressing medically refractory CRS. ${ }^{10}$ Nevertheless, different efforts to develop minimally invasive techniques to achieve the same goals of increased sinus ventilation, improved topical drug delivery and avoidance of mucosal damage have been developed. ${ }^{8}$

Our experience and results suggest that the placement of LDDs for patients with CRSsNP is an achievable and safe intervention. The learning curve of this procedure is relatively fast, and adequate placement was achieved in all patients. We found a clinically and statistically significant improvement 1 month after surgery in the SNOT-20 score of all patients. Although most of our patients used the stent for $>1$ month, the score was only assessed in all of them 1 month after the surgery, and the following revisions differed significatively in time in all of them, and not all of them were reassessed with the questionnaire; for this reason, we could only evaluate them objectively in this single period of time. However the ideal duration for stents and drug-eluting stents in sinus surgery has not been defined. ${ }^{11}$ Most investigators recommend the use of stents for at least 6 weeks, but there have been some authors who describe prolonged stenting for years. ${ }^{12,13}$

A previous cadaver study had proven that LDDs can be used as a potential alternate technique for the delivery of sinus irrigation. ${ }^{9}$ To our knowledge, there are not any studies published yet in the literature describing the outcomes of this technique in patients, although Nayak et al, in a poster session presented at the Combined Otolaryngology Spring Meeting in April 2018, ${ }^{14}$ described 23 patients with symptoms of sinusitis/CRS who received bilateral LDDs under conscious sedation in 2 different institutions. The SNOT-20 score declined significantly at day 7 and at day 30 in one institution, and at week 4 and at week 8 in the other institution. The Lund-Mackay radiographic score was also assessed in this last group, also presenting a significant improvement. The author has also described some locoregional complications, including foreign body sensation, preseptal inflammation, and device occlusion in a minority of cases that were self-limited.

Minimally invasive techniques can be categorized as MISTs, such as Ethmoid punch sinusotomy and balloon catheter dilatation (BCD) sinusotomy. ${ }^{7,8}$ We understand that LDDs could be considered as a MIST. This technique can improve topical irrigation and re-establish the nasal ventilation in patients with CRS with ethmoid sinus compromise, where topical nasal treatment has limited penetration. Furthermore, it can be considered as a less invasive rhinologic procedure for the treatment of ethmoid sinusitis. It also has advantages over FESS, such as a lower risk of mucosal stripping, which may result in sinus bone exposure and, eventually, in scarring and in the recrudescence of the CRS disease. ${ }^{15}$ It can also be rapidly performed when compared with FESS, does not require advanced training in endoscopic sinus surgery, and can be performed in-office under local anesthesia or sedation. Minimally invasive techniques had demonstrated short-term outcomes comparable with FESS, ${ }^{16}$ but the evidence of supporting the use of these techniques is limited and longer outcomes remain controversial. ${ }^{17}$

The main limitation of the present study is that the outcome of LDD placement and, therefore, the reduction in the SNOT-20 score cannot be fully separated from the effects of an additional turbinoplasty, septoplasty, or another sinus procedure performed in the patients. Long-term outcomes need to be assessed, and studies in larger populations with comparative groups might provide a better support for this new technique.

\section{Conclusion}

The use of LDDs (Sinopsys Lacrimal Stent) is a novel and feasible technique that is part of this new wave of less invasive rhinologic procedures for the treatment of CRS that do not respond to medical treatment. It can also be considered a less invasive alternative for the treatment of the ethmoid sinus, which is frequently involved with inflammatory disease and exacerbations of the symptoms. This novel approach can be performed in-office with minimal disruption of the local mucosa, and improve topical irrigation and medication of the ethmoid sinus.

\section{Conflicts of Interest}

Dr. Peter M Baptista served during 2017 up to February 2018 as a consultant for Sinopsys. Has no financial relationship with them at this moment. Sinospsys has not been approved or cleared for use with a drug or medication to treat CRS in any global market.

\section{References}

1 A Fokkens WJ, Lund VJ, Mullol J, et al. European Position Paper on Rhinosinusitis and Nasal Polyps 2012. Rhinol Suppl 2012;(23): $1-298$ 
2 Orlandi RR, Kingdom TT, Hwang PH. International Consensus Statement on Allergy and Rhinology: Rhinosinusitis Executive Summary. Int Forum Allergy Rhinol 2016;6(Suppl 1): S3-S21

3 Mace J, Michael YL, Carlson NE, Litvack JR, Smith TL. Effects of depression on quality of life improvement after endoscopic sinus surgery. Laryngoscope 2008;118(03):528-534

4 Bhattacharyya $\mathrm{N}$. Incremental health care utilization and expenditures for chronic rhinosinusitis in the United States. Ann Otol Rhinol Laryngol 2011;120(07):423-427

5 Senior BA, Kennedy DW, Tanabodee J, Kroger H, Hassab M, Lanza D. Long-term results of functional endoscopic sinus surgery. Laryngoscope 1998;108(02):151-157

6 Harvey RJ, Goddard JC, Wise SK, Schlosser RJ. Effects of endoscopic sinus surgery and delivery device on cadaver sinus irrigation. Otolaryngol Head Neck Surg 2008;139(01):137-142

7 Catalano PJ, Strouch M. The minimally invasive sinus technique: theory and practice. Otolaryngol Clin North Am 2004;37(02): 401-409, viii.

8 Velasquez N, Thamboo A, Abuzeid WM, Nayak JV. Safe treatment of ethmoid sinusitis utilizing minimally invasive ethmoid punch sinusotomy in chronic rhinosinusitis without polyposis patients. Laryngoscope 2017;127(06):1268-1275

9 Tajudeen BA, Gupta S, Willoughby B, Kennedy DW. Delivering irrigation to the anterior ethmoid region: evaluation of a lacrimal diversion device in a cadaver model. Int Forum Allergy Rhinol 2017;7(08):794-800
10 Smith TL, Batra PS, Seiden AM, Hannley M. Evidence supporting endoscopic sinus surgery in the management of adult chronic rhinosinusitis: a systematic review. Am J Rhinol 2005;19(06):537-543

11 Hauser LJ, Turner JH, Chandra RK. Trends in the Use of Stents and Drug-Eluting Stents in Sinus Surgery. Otolaryngol Clin North Am 2017;50(03):565-571

12 Weber R, Mai R, Hosemann W, Draf W, Toffel P. The success of 6month stenting in endonasal frontal sinus surgery. Ear Nose Throat J 2000;79(12):930-932, 934, 937-938 passim

13 Orlandi RR, Knight J. Prolonged stenting of the frontal sinus. Laryngoscope 2009;119(01):190-192

14 NayakJV, Thamboo A, Gall P, Kennedy DW. Evidence of symptomatic and radiographic improvement following conjunctivodacrocystosinusotomy (CDCS) with lacrimal diversion device (LDD) placement as a novel therapeutic platform for sinusitis. Poster session presented at: Combined Otolaryngology Spring Meeting.2018 April 18-22; Maryland, USA

15 Ramakrishnan VR, Kingdom TT, Nayak JV, Hwang PH, Orlandi RR. Nationwide incidence of major complications in endoscopic sinus surgery. Int Forum Allergy Rhinol 2012;2(01):34-39

16 Catalano P, Roffman E. Outcome in patients with chronic sinusitis after the minimally invasive sinus technique. Am J Rhinol 2003;17 (01):17-22

17 Cutler J, Bikhazi N, Light J, Truitt T, Schwartz M; REMODEL Study Investigators. Standalone balloon dilation versus sinus surgery for chronic rhinosinusitis: a prospective, multicenter, randomized, controlled trial. Am J Rhinol Allergy 2013;27(05):416-422 